J. Nonlinear Var. Anal. 2 (2018), No. 2, pp. 123-130

Available online at http://jnva.biemdas.com

https://doi.org/10.23952/jnva.2.2018.2.02

\title{
INTRIGUING MAXIMALLY MONOTONE OPERATORS DERIVED FROM NONSUNNY NONEXPANSIVE RETRACTIONS
}

\author{
HEINZ H. BAUSCHKE ${ }^{1, *}$, LEVI MILLER, WALAA M. MOURSI ${ }^{2,3}$ \\ ${ }^{1}$ Mathematics, University of British Columbia, Kelowna, B.C. V1V 1V7, Canada \\ ${ }^{2}$ Electrical Engineering, Stanford University, Stanford, CA 94305, USA \\ ${ }^{3}$ Mathematics Department, Faculty of Science, Mansoura University, Mansoura 35516, Egypt
}

\begin{abstract}
Monotone operator theory and fixed point theory for nonexpansive mappings are central areas in modern nonlinear analysis and optimization. Although these areas are fairly well developed, almost all examples published are based on subdifferential operators, linear relations, or combinations thereof. In this paper, we construct an intriguing maximally monotone operator induced by a certain nonexpansive retraction. We analyze this operator, which does not appear to be assembled from subdifferential operators or linear relations, in some detail. Particular emphasis is placed on duality and strong monotonicity. Keywords. Firmly nonexpansive mapping; Maximally monotone operator; Nonexpansive mapping; Resolvent; Retraction.
\end{abstract}

2010 Mathematics Subject Classification. 47H05, 47H09, 90C25.

\section{INTRODUCTION}

Suppose that

$X$ is a real Hilbert space,

with inner product $\langle\cdot, \cdot\rangle$ and induced norm $\|\cdot\|$. Throughout, we assume that $X \neq\{0\}$ and that

$$
e \in X \text { and }\|e\| \leq 1 \text {. }
$$

Now define

$$
R_{e}: X \rightarrow X: x \mapsto\|x\| \cdot e .
$$

The mapping $R_{e}$ is nonexpansive and thus induces an associated firmly nonexpansive mapping $T_{e}$ as well as a maximally monotone operator $A_{e}$.

The goal of this paper is to present fundamental properties of $R_{e}, T_{e}$, and $A_{e}$. These operators are neither subdifferential operators nor linear relations; consequently, they provide a new testing ground for properties in monotone operator theory and fixed point theory.

The paper is organized as follows. In Section 2, we focus on $R_{e}$, the induced firmly nonexpansive mapping $T_{e}$ and the maximally monotone operator $A_{e}$. Duality and stronger notions of monotonicity are considered in Section 3. We conclude the paper in Section 4 with a discussion on the resolvent iteration and the resolvent average.

\footnotetext{
${ }^{*}$ Corresponding author.

E-mail addresses: heinz.bauschke@ubc.ca (H.H. Bauschke), levi@levimiller.ca (L. Miller), wmoursi@stanford.edu (W.M. Moursi).

Received May 23, 2018; Accepted June 9, 2018.
}

(C)2018 Journal of Nonlinear and Variational Analysis 
The notation employed is standard and follows, e.g., [2]. Finally, we assume the reader is familiar with basic monotone operator theory and fixed point theory, as can be found in e.g., [2], [4], [5], [6], [7], [11], [12], [13], [14], [15], [16], or [17].

\section{2. $R_{e}, T_{e}, \mathrm{AND} A_{e}$}

We start by collecting some properties of $R_{e}$. Item 1 of the following result states that $\mathbb{R}_{+} \cdot e$ is a nonexpansive retract of $X$, via $R_{e}$. (See [9] for more on nonexpansive retracts.)

Proposition 2.1. (l) If $\|e\|=1$, then $R_{e}$ is a nonexpansive retraction of $\mathbb{R}_{+} \cdot e$ and $R_{e}$ is not a Banach contraction.

(2) If $\|e\|<1$, then $R_{e}$ is a Banach contraction with optimal Lipschitz constant $\|e\|$ and Fix $R_{e}=\{0\}$.

(3) If $0<\|e\| \leq 1$ and $f \in\{e\}^{\perp}$ with $\|f\|=1$, then $R_{e}$ is not sunny.

(4) $R_{e}$ is nonexpansive.

Proof. We have

$$
(\forall x \in X)(\forall y \in X) \quad\left\|R_{e} x-R_{e} y\right\|=\|\| x\|e-\| y\|e\|=|\|x\|-\|y\|| \cdot\|e\| \leq\|e\| \cdot\|x-y\|,
$$

which shows that $R_{e}$ is Lipschitz continuous with constant $\|e\|$; moreover,

$$
\left(\forall x \in \mathbb{R}_{+} \cdot e\right)\left(\forall y \in \mathbb{R}_{+} \cdot e\right) \quad\left\|R_{e} x-R_{e} y\right\|=\|e\| \cdot\|x-y\| .
$$

It is clear that Fix $R_{e} \subseteq \operatorname{ran} R_{e}=\mathbb{R}_{+} e$. If $x \in \mathbb{R}_{+} \cdot e$, say $x=\rho e$, where $\rho \in \mathbb{R}_{+}$, then $R_{e} x=\|x\| e=$ $\|\rho e\| e=\rho\|e\| e=\|e\| x$. Hence

$$
(\forall x \in X) \quad x=R_{e} x \Leftrightarrow[x=0 \text { or }\|e\|=1] .
$$

1: In view of (2.1) and the assumption that $\|e\|=1$, it is clear that $R_{e}$ is nonexpansive. From (2.3) we deduce that Fix $R_{e}=\mathbb{R}_{+} \cdot e$; thus, $R_{e}$ is a nonexpansive retract of $\mathbb{R}_{+} \cdot e$.

2: Combine (2.1) with (2.3).

3: Set $x=\|e\|^{-1} e+\sqrt{3} f$. Then $\|x\|^{2}=1+3=4$ and so $R_{e} x=\|x\| e=2 e$. Now consider

$$
y=\left(x+R_{e} x\right) / 2=2^{-1}\left(2+\|e\|^{-1}\right) e+2^{-1} \sqrt{3} f \in\left[x, R_{e} x\right] .
$$

Then

$$
\|y\|^{2}=\left(2^{-1}\left(2+\|e\|^{-1}\right)\right)^{2}\|e\|^{2}+\left(2^{-1} \sqrt{3}\right)^{2}=1+\|e\|+\|e\|^{2} \leq 3<4=\|x\|^{2} .
$$

We deduce that $R_{e} x=\|x\| e \neq\|y\| e=R_{e} y=\|y\| e$ and thus $R_{e}$ is not sunny.

4: Combine 1 and 2.

Proposition 2.14 shows that

$$
T_{e}: X \rightarrow X: x \mapsto \frac{1}{2} x+\frac{1}{2} R_{e} x=\frac{1}{2} x+\frac{1}{2}\|x\| e
$$

is firmly nonexpansive and hence the resolvent $J_{A_{e}}=\left(\mathrm{Id}+A_{e}\right)^{-1}$ of the maximally monotone operator

$$
A_{e}=T_{e}^{-1}-\mathrm{Id} .
$$

Our next task is to provide an explicit formula for $A_{e}$. It turns out that $A_{e}$ behaves quite differently, depending on whether $\|e\|=1$ or $\|e\|<1$. 
INTRIGUING MAXIMALLY MONOTONE OPERATORS DERIVED FROM NONSUNNY NONEXPANSIVE RETRACTIONS 125

Theorem $2.1\left(A_{e}\right.$ for $\left.\|e\|=1\right)$. Suppose that $\|e\|=1$. Then the maximally monotone operator $A_{e}$ is given by

$$
(\forall x \in X) \quad A_{e} x= \begin{cases}x-\frac{\|x\|^{2}}{\langle e, x\rangle} \cdot e, & \text { if }\langle e, x\rangle>0 ; \\ \mathbb{R}_{-} \cdot e, & \text { if } x=0 ; \\ \varnothing, & \text { otherwise. }\end{cases}
$$

Proof. Denote the right-hand side of (2.5) by $B$, i.e., set

$$
(\forall x \in X) \quad B x= \begin{cases}x-\frac{\|x\|^{2}}{\langle e, x\rangle} \cdot e, & \text { if }\langle e, x\rangle>0 ; \\ \mathbb{R}_{-} \cdot e, & \text { if } x=0 ; \\ \varnothing, & \text { otherwise. }\end{cases}
$$

Our job is to show that $B=A_{e}$, and for that it suffices to show that gra $B=\operatorname{gra} A_{e}=\left\{\left(T_{e} x, x-T_{e} x\right) \mid x \in X\right\}$ by the Minty parametrization [10]. For convenience, we also write $T$ instead of $T_{e}$.

First, let $x \in X$. We need to consider two cases.

Case 1: $\langle e, x\rangle>0$.

Set $u=x-\|x\|^{2}\langle e, x\rangle^{-1} e \in B x$ and $y=x+u=2 x-\|x\|^{2}\langle e, x\rangle^{-1} e$. Then

$$
\|u\|^{2}=\|x\|^{2}+\|x\|^{4}\langle e, x\rangle^{-2}\|e\|^{2}-2\|x\|^{2}\langle e, x\rangle^{-1}\langle x, e\rangle=\|x\|^{4}\langle e, x\rangle^{-2}-\|x\|^{2}
$$

and

$$
\langle x, u\rangle=\left\langle x, x-\|x\|^{2}\langle e, x\rangle^{-1} e\right\rangle=\langle x, x\rangle-\|x\|^{2}\langle e, x\rangle^{-1}\langle x, e\rangle=0 .
$$

Hence

$$
\|y\|^{2}=\|x\|^{2}+\|u\|^{2}+2\langle x, u\rangle=\|x\|^{2}+\left(\|x\|^{4}\langle e, x\rangle^{-2}-\|x\|^{2}\right)+2 \cdot 0=\|x\|^{4}\langle e, x\rangle^{-2}
$$

and thus $\|y\|=\|x\|^{2}\langle e, x\rangle^{-1}$. Hence

$$
\begin{aligned}
T y & =\frac{1}{2} y+\frac{1}{2}\|y\| e=\frac{1}{2}(x+u)+\frac{1}{2}\|x\|^{2}\langle e, x\rangle^{-1} e \\
& =\frac{1}{2}\left(x+\left(x-\|x\|^{2}\langle e, x\rangle^{-1} e\right)+\|x\|^{2}\langle e, x\rangle^{-1} e\right) \\
& =x
\end{aligned}
$$

and

$$
y-T y=(x+u)-x=u .
$$

We have shown that $(x, u)=(T y, y-T y) \in \operatorname{gra} A_{e}$ as required.

Case 2: $x=0$.

Here we set $u=\eta e$, where $\eta \leq 0$, and again $y=x+u=\eta e$. Then

$$
\|y\|=\|u\|=\|\eta e\|=|\eta| \cdot\|e\|=|\eta|=-\eta .
$$

Hence

$$
T y=\frac{1}{2} y+\frac{1}{2}\|y\| e=\frac{1}{2} \eta e+\frac{1}{2}(-\eta) e=0=x
$$

and $y-T y=\eta e-x=u-0=u$. Again, we have shown that $(x, u)=(T y, y-T y) \in \operatorname{gra} A_{e}$, as claimed.

Combining Case 1 and Case 2, we obtain the conclusion

$$
\operatorname{gra} B \subseteq \operatorname{gra} A_{e} .
$$


Conversely, let $y \in X$. Set $x=T y=\frac{1}{2} y+\frac{1}{2}\|y\| e$ and note that $y-T y=\frac{1}{2} y-\frac{1}{2}\|y\| e$. Furthermore,

$$
2\langle e, x\rangle=\langle y, e\rangle+\|y\|\langle e, e\rangle=\langle y, e\rangle+\|y\| \geq-\|y\| \cdot\|e\|+\|y\|=0
$$

with equality if and only if $y \in \mathbb{R}_{-} \cdot e$, i.e., $x=0$, by Cauchy-Schwarz. Moreover,

$$
\|x\|^{2}=\left\|\frac{1}{2} y+\frac{1}{2}\right\| y\|e\|^{2}=\frac{1}{4}\|y\|^{2}+\frac{1}{4}\|y\|^{2}+\frac{1}{2}\|y\|\langle y, e\rangle=\frac{1}{2}\left(\|y\|^{2}+\|y\|\langle y, e\rangle\right)
$$

and

$$
\langle e, x\rangle=\left\langle e, \frac{1}{2} y+\frac{1}{2}\|y\| e\right\rangle=\frac{1}{2}\langle e, y\rangle+\frac{1}{2}\|y\| .
$$

We now consider two conceivable alternatives.

Case 1: $\langle e, x\rangle>0$.

Then

$$
\begin{aligned}
B x & =x-\frac{\|x\|^{2}}{\langle e, x\rangle} \cdot e=\frac{1}{2} y+\frac{1}{2}\|y\| e-\frac{\frac{1}{2}\left(\|y\|^{2}+\|y\|\langle y, e\rangle\right)}{\frac{1}{2}\langle e, y\rangle+\frac{1}{2}\|y\|} \cdot e \\
& =\frac{1}{2} y-\frac{1}{2}\|y\| e \\
& =y-T y .
\end{aligned}
$$

Therefore, $(T y, y-T y)=(x, B x) \in \operatorname{gra} B$.

Case 2: $x=0$.

Then $y=\eta e$, where $\eta \in \mathbb{R}_{-}$. Thus

$$
y-T y=\frac{1}{2} y-\frac{1}{2}\|y\| e=\frac{1}{2} \eta e-\frac{1}{2}|\eta| e=\eta e \in B 0=B x .
$$

Therefore, $(y, T y) \in\{0\} \times B 0 \subseteq$ gra $B$.

Combining Case 1 with Case 2, we deduce that

$$
\operatorname{gra} A_{e} \subseteq \operatorname{gra} B .
$$

Finally, (2.11) and (2.13) yield the result.

Theorem $2.2\left(A_{e}\right.$ for $\left.\|e\|<1\right)$. Suppose that $\|e\|<1$. Then the maximally monotone operator $A_{e}$ is given by

$$
(\forall x \in X) \quad A_{e} x=x+\frac{2\langle e, x\rangle-2 \sqrt{\left(1-\|e\|^{2}\right)\|x\|^{2}+\langle e, x\rangle^{2}}}{1-\|e\|^{2}} \cdot e .
$$

Proof. Denote the right-hand side of (2.14) by $B$, i.e., set

$$
(\forall x \in X) \quad B x=x+\frac{2\langle e, x\rangle-2 \sqrt{\left(1-\|e\|^{2}\right)\|x\|^{2}+\langle e, x\rangle^{2}}}{1-\|e\|^{2}} \cdot e .
$$

We also write

$$
B x=x+\rho(x) \cdot e
$$

and observe that $\rho(x)$ is the nonpositive root of the quadratic equation

$$
\left(1-\|e\|^{2}\right) \rho^{2}-4\langle e, x\rangle \rho-4\|x\|^{2}=0 .
$$

Once again, our job is to show that $B=A_{e}$, and for that it suffices to show that $\operatorname{gra} B=\operatorname{gra} A_{e}=\left\{\left(T_{e} x, x-\right.\right.$ $\left.\left.T_{e} x\right) \mid x \in X\right\}$ by the Minty parametrization [10]. For convenience, we also abbreviate $T=T_{e}$ and $\rho=\rho(x)$. 
INTRIGUING MAXIMALLY MONOTONE OPERATORS DERIVED FROM NONSUNNY NONEXPANSIVE RETRACTIONS 127

First, let $x \in X$ and set $y=x+B x=2 x+\rho e$. Then $\|y\|^{2}=\|2 x+\rho e\|^{2}=4\|x\|^{2}+4 \rho\langle x, e\rangle+\rho^{2}\|e\|^{2}=$ $4\|x\|^{2}+4 \rho\langle x, e\rangle+\rho^{2}\left(\|e\|^{2}-1\right)+\rho^{2}=0+\rho^{2}=\rho^{2}$ by (2.17); thus, $\|y\|=|\rho|=-\rho$. Hence

$$
T y=\frac{1}{2} y+\frac{1}{2}\|y\| e=\frac{1}{2}(2 x+\rho e)+\frac{1}{2}(-\rho) e=x
$$

and so

$$
y-T y=(2 x+\rho e)-x=x+\rho e=B x .
$$

It follows that $(x, B x)=(T y, y-T y) \in \operatorname{gra} A_{e}$ and thus

$$
\operatorname{gra} B \subseteq \operatorname{gra} A_{e} .
$$

Conversely, let $y \in X$, set $x=T y=\frac{1}{2} y+\frac{1}{2}\|y\| e$ and note that $y-T y=\frac{1}{2} y-\frac{1}{2}\|y\| e$. We have

$$
\|x\|^{2}=\left\|\frac{1}{2} y+\frac{1}{2}\right\| y\|e\|^{2}=\frac{1}{4}\|y\|^{2}+\frac{1}{4}\|y\|^{2}\|e\|^{2}+\frac{1}{2}\|y\|\langle y, e\rangle
$$

and $\langle e, x\rangle=\left\langle e, \frac{1}{2} y+\frac{1}{2}\|y\| e\right\rangle=\frac{1}{2}\langle e, y\rangle+\frac{1}{2}\|y\|\|e\|^{2}$. Hence

$$
\begin{aligned}
& \left(1-\|e\|^{2}\right)(-\|y\|)^{2}-4\langle e, x\rangle(-\|y\|)-4\|x\|^{2} \\
& =\left(1-\|e\|^{2}\right)\|y\|^{2}+2\|y\|\langle e, y+\|y\| e\rangle-\left(\|y\|^{2}+\|y\|^{2}\|e\|^{2}+2\|y\|\langle y, e\rangle\right) \\
& =0 .
\end{aligned}
$$

In view of (2.17) and the fact that $-\|y\| \leq 0$, it follows that $-\|y\|=\rho(x)$. Hence

$$
B x=x+\rho(x) e=\left(\frac{1}{2} y+\frac{1}{2}\|y\| e\right)-\|y\| e=\frac{1}{2} y-\frac{1}{2}\|y\| e=y-T y .
$$

Hence $(T y, y-T y)=(x, B x) \in \operatorname{gra} B$ and thus

$$
\operatorname{gra} A_{e} \subseteq \operatorname{gra} B .
$$

The conclusion now follows by combining (2.20) with (2.23).

\section{DUALITY AND STRONGER NOTIONS OF MONOTONICITY}

If $A: X \rightrightarrows X$ is maximally monotone, then its $d u a l$ operator is $A^{-1}$, and the corresponding dual objects of the resolvent and reflected resolvent are $\mathrm{Id}-J_{A}$ and $-R_{A}$, respectively [3]. We now identify the dual objects, which have pleasant explicit formulae, as well as some other interesting properties.

Lemma 3.1. Recall that $A_{e}, T_{e}$, and $R_{e}$ are defined in (2), (2), and (1), respectively. Then the following hold:

(1) (duality) $-R_{e}=R_{-e}$, Id $-T_{e}=T_{-e}$, and $\left(A_{e}\right)^{-1}=A_{-e}$.

(2) (cone) $(\forall x \in X)\left(\forall \lambda \in \mathbb{R}_{++}\right) A_{e}(\lambda x)=\lambda A_{e} x$. Consequently, gra $A$ is a (nonconvex) cone.

(3) If $\|e\|=1$, then $\left(\forall(x, u) \in \operatorname{gra} A_{e}\right)\langle x, u\rangle=0$.

(4) If $\|e\|=1$, then zer $A_{e}=\left(A_{e}\right)^{-1}(0)=\mathbb{R}_{+} \cdot e$.

Proof. 1: It is clear that $R_{\left(A_{e}\right)^{-1}}=-R_{A_{e}}=-R_{e}=-\|\cdot\| e=R_{-e}$. Hence, $\left(A_{e}\right)^{-1}=A_{-e}$. Consequently, by the inverse resolvent identity and (2), we have $\mathrm{Id}-T_{e}=\mathrm{Id}-J_{A_{e}}=J_{\left(A_{e}\right)^{-1}}=J_{A_{-e}}=T_{-e}$. 2: This can be directly verified using (2.5) and (2.14). 3: Let $x \in \operatorname{dom} A$. If $x=0$ then $\left(\forall u \in A x=\mathbb{R}_{-} \cdot e\right)$ we have $\langle x, u\rangle=0$. Now suppose that $\langle e, x\rangle>0$. Then $A x$ is a singleton and $\langle x, A x\rangle=\left\langle x, x-\frac{\|x\|}{\langle e, x\rangle} \cdot e\right\rangle=\|x\|-\|x\|=$ 0. 4: Indeed, zer $A_{e}=A_{e}^{-1}(0)=A_{-e}(0)=\mathbb{R}_{-} \cdot(-e)=\mathbb{R}_{+} \cdot e$. 
Strong monotonicity, paramonotonicity, cocoercivity, and $3^{*}$ monotonicity are perhaps the most important properties a maximally monotone operators can have. The following two results provide a complete characterization of these properties. Once again, the norm $\|e\|$ plays a crucial role.

Proposition 3.1. Suppose that $\|e\|=1$ and that $X$ is not one-dimensional. Then the following hold:

(1) $A_{e}$ is not paramonotone. Consequently, $A_{e}$ is neither strictly nor strongly monotone.

(2) $A_{e}$ is not $3^{*}$ monotone.

Proof. 1: Let $\alpha \in] 1,+\infty\left[\right.$, let $x \in \operatorname{dom} A \backslash \mathbb{R}_{+} \cdot e$ and set $y=\alpha x$. Note that $\langle e, x\rangle>0$, that $A y=A(\alpha x)=$ $\alpha A x \neq 0$ by Lemma $3.12 \& 4$, and that $A x$ (and consequently $A y$ ) is a singleton. Therefore, by Lemma 3.13 $\langle x-y, A x-A y\rangle=\langle(1-\alpha) x,(1-\alpha) A x\rangle=(1-\alpha)^{2}\langle x, A x\rangle=0$. However, $(x, A y) \notin \operatorname{gra} A$, hence $A$ is not paramonotone.

2: Indeed, let $\alpha>0$, let $f \in\{e\}^{\perp}$ such that $\|f\|=1$, set $x=2(f-e)$, set $y=0$ and set $z=2 \alpha f$. We also write $T$ instead of $T_{e}$ for convenience. Now

$$
\begin{aligned}
\langle T x-T z,(\mathrm{Id}-T) y-(\mathrm{Id}-T) z\rangle & =-\langle T x-T z,(\mathrm{Id}-T) z\rangle \\
& =-\langle f-e+\|f-e\| e-\alpha(f+e), \alpha(f-e)\rangle \\
& =-\langle f-e+\|f-e\| e, \alpha(f-e)\rangle+\alpha^{2}\left(\|f\|^{2}-\|e\|^{2}\right) \\
& =-\alpha\langle f-e+\|f-e\| e, f-e\rangle \\
& =-\alpha\left(\|f-e\|^{2}-\|f-e\|^{2}\right)=-\alpha(2-\sqrt{2}) .
\end{aligned}
$$

Therefore, we conclude that

$$
\inf _{z \in X}\langle T x-T z,(\mathrm{Id}-T) y-(\mathrm{Id}-T) z\rangle=-\infty .
$$

Hence $A$ is not $3^{*}$ monotone by [3, Theorem 2.1(xvii)].

Proposition 3.2. Suppose that $\|e\|<1$. Then the following hold:

(1) $A_{e}$ is strongly monotone, with sharp constant $\frac{1-\|e\|}{1+\|e\|}$.

(2) $A_{e}$ is cocoercive, with sharp constant $\frac{1-\|e\|}{1+\|e\|}$.

(3) $A_{e}$ is paramonotone.

(4) $A_{e}$ is $3^{*}$ monotone.

(5) $A_{e}$ is a displacement map if and only if $\|e\| \leq \frac{1}{3}$.

Proof. 1: Let $(x, y) \in X \times X$. By the Minty parametrization [10] of $\operatorname{gra} A_{e}(\exists(u, v) \in X \times X)$ such that $(x, A x)=\left(T_{e} u, u-T_{e} u\right)=\frac{1}{2}(u+\|u\| e, u-\|u\| e)$ and $(y, A y)=\left(T_{e} v, v-T_{e} v\right)=\frac{1}{2}(v+\|v\| e, v-\|v\| e)$. Now, on the one hand, by the reverse triangle inequality we have

$$
\begin{aligned}
4\langle x-y, A x-A y\rangle & =\langle(u-v)+(\|u\| e-\|v\| e),(u-v)-(\|u\| e-\|v\| e)\rangle \\
& =\|u-v\|^{2}-(\|u\|-\|v\|)^{2}\|e\|^{2} \geq\left(1-\|e\|^{2}\right)\|u-v\|^{2} .
\end{aligned}
$$

On the other hand, using the triangle inequality and the reverse triangle inequality we have

$$
2\|x-y\|=\|(u-v)+(\|u\|-\|v\|) e\| \leq\|u-v\|+|\|u\|-\|v\||\|e\| \leq(1+\|e\|)\|u-v\| .
$$

Combining (3.2) and (3.3) yields

$$
\left\langle x-y, A x-A y\left\langle\geq \frac{1-\|e\|^{2}}{(1+\|e\|)^{2}}\|x-y\|^{2}=\frac{1-\|e\|}{1+\|e\|}\|x-y\|^{2} .\right.\right.
$$


INTRIGUING MAXIMALLY MONOTONE OPERATORS DERIVED FROM NONSUNNY NONEXPANSIVE RETRACTIONS 129

To show that the strong monotonicity constant is sharp we set $(x, y)=(e, 0)$. Now

$$
\begin{aligned}
\langle e-0, A e-A 0\rangle=\langle e, A e\rangle & =\left\langle e,\left(1+\frac{2\langle e, e\rangle-2 \sqrt{\left(1-\|e\|^{2}\right)\|e\|^{2}+\langle e, e\rangle^{2}}}{1-\|e\|^{2}}\right) e\right\rangle \\
& =\left(1+\frac{2\|e\|^{2}-2\|e\|}{1-\|e\|^{2}}\right)\|e\|^{2}=\left(\frac{\|e\|^{2}-2\|e\|+1}{1-\|e\|^{2}}\right)\|e\|^{2} \\
& =\frac{1-\|e\|}{1+\|e\|}\|e-0\|^{2} .
\end{aligned}
$$

2: It follows from 1 applied with $e$ replaced by $-e$ that $A_{-e}$ is strongly monotone with sharp constant $\frac{1-\|e\|}{1+\|e\|}$. Combining with 1 and [2, Example 22.7] we conclude that $A_{e}=\left(A_{-e}\right)^{-1}$ is $\frac{1-\|e\|}{1+\|e\|}$-cocoercive. 3: Combine 2 and [2, Example 22.8]. 4: Combine 2 and [2, Example 25.20(i)]. 5: It follows from [1, Proposition 4.13] and 2 in view of [2, Example 22.7] that $A_{e}$ is a displacement map if and only if $\frac{1-\|e\|}{1+\|e\|} \geq \frac{1}{2}$; equivalently $\|e\| \leq \frac{1}{3}$.

Remark 3.1 (the one-dimensional case for $\|e\|=1$ ). Now suppose that $X$ is one-dimensional. Then $A_{e}$ is a subdifferential operator; in fact, $A_{e}=N_{\mathbb{R}_{+} e}$. Hence $A_{e}$ is both paramonotone (see, e.g., [2, Example 22.4(i)]) and $3^{*}$ monotone (see, e.g., [2, Example 25.14]), but neither strictly nor strongly monotone.

Remark 3.2 (cyclic monotonicity). If $X$ is one-dimensional or $e=0$, then $A_{e}$ is clearly cyclically monotone; otherwise, $A_{e}$ is not a gradient by the formulae provided in Section 2. Based on some numerical experiments, we conjecture that the degree of cyclic monotonicity decreases from $+\infty$ (when $\|e\|=0$ ) to 2 (when $\|e\|=1$ ) as $\|e\|$ increases; however, we do not know at which values the transitions occur.

\section{RESOlVENT ITERATION AND AVERAGE}

It is well known that the sequence $\left(\left(T_{e}\right)^{n} x_{0}\right)_{n \in \mathbb{N}}$ converges weakly to some point in Fix $T_{e}=\operatorname{zer} A_{e}$; however, more can be said in our setting:

Proposition 4.1 (resolvent iteration). Suppose that $X$ is not one-dimensional and let $x_{0} \in X$. The following hold true:

(1) $\left(\left(T_{e}\right)^{n} x_{0}\right)_{n \in \mathbb{N}}$ converges strongly to some point in Fix $T_{e}=\operatorname{zer} A_{e}$.

(2) If $\|e\|<1$, then $\left(T_{e}\right)^{n} x_{0} \rightarrow 0$ linearly, with rate $(1+\|e\|) / 2$.

(3) If $\|e\|=1$, then $\left(T_{e}\right)^{n} x_{0} \rightarrow\left\|x_{0}\right\| \operatorname{sinc}\left(\theta_{0}\right) e$, where sinc is the (unnormalized) sinc function and $\theta_{0} \in[0, \pi]$ satisfies $\cos \left(\theta_{0}\right)\left\|x_{0}\right\|=\left\langle x_{0}, e\right\rangle$.

Proof. 1: On the one hand, the convergence is known to be weak, see, e.g., [2, Proposition 5.16(iii)]. On the other hand, all iterates lie in $\operatorname{span}\left\{e, x_{0}\right\}$. Altogether, the convergence must be strong.

2\&3: Write $x_{n}=\left(T_{e}\right)^{n} x_{0}$ for every $n \in \mathbb{N}$. The result is clear when $e=0$ since then $T_{e}=\frac{1}{2} \mathrm{Id}$. Assume that $e \neq 0$ and that $x_{0} \neq 0$, and let us work in "polar coordinates", i.e., pick $f \in \operatorname{span}\left\{x_{0}, e\right\} \cap\{e\}^{\perp}$ such that $\|f\|=1$ and write $x_{n}=\left\|x_{n}\right\|\left(\cos \left(\theta_{n}\right) \widehat{e}+\sin \left(\theta_{n}\right) f\right)$, where $\widehat{e}=e /\|e\|$ and $\theta_{n} \in[0, \pi]$. Then

$$
\left\|x_{n+1}\right\|^{2} /\left\|x_{n}\right\|^{2}=\left(1+2 \cos \left(\theta_{n}\right)\|e\|+\|e\|^{2}\right) / 4
$$

and $\theta_{n} \rightarrow 0^{+}$. Thus $\left\|x_{n+1}\right\|^{2} /\left\|x_{n}\right\|^{2} \rightarrow\left(1+\|e\|^{2}+2\|e\|\right) / 4=((1+\|e\|) / 2)^{2}$ and the result follows when $\|e\|<1$.

Now assume that $\|e\|=1$. Then, by (4.1), one has

$$
\left\|x_{n+1}\right\|^{2}=\left\|x_{n}\right\|^{2}\left(1+\cos \left(\theta_{n}\right)\right) / 2=\left\|x_{n}\right\|^{2} \cos ^{2}\left(\theta_{n} / 2\right) .
$$


Inductively, it follows that

$$
\begin{aligned}
x_{n} & =\left\|x_{n}\right\|\left(\cos \left(\theta_{0} / 2^{n}\right) e+\sin \left(\theta_{0} / 2^{n}\right) f\right) \\
& =\left\|x_{0}\right\|\left(\prod_{k=1}^{n-1} \cos \left(\theta_{0} / 2^{k}\right)\right)\left(\cos \left(\theta_{0} / 2^{n}\right) e+\sin \left(\theta / 2^{n}\right) f\right) \\
& \rightarrow\left\|x_{0}\right\|\left(\prod_{k=1}^{\infty} \cos \left(\theta_{0} / 2^{k}\right)\right) e \\
& =\left\|x_{0}\right\| \operatorname{sinc}\left(\theta_{0}\right) e,
\end{aligned}
$$

where we use [8, equation (1.3) on page 2] in (4.2d).

We conclude this paper with an observation on the resolvent average that follows readily from the definition.

Proposition 4.2 (resolvent average). Suppose that $\lambda_{1}, \lambda_{2}, \ldots, \lambda_{m}$ are in $[0,1]$ such that $\sum_{i=1}^{m} \lambda_{i}=1$ and that $e_{1}, \ldots, e_{m}$ are in the unit ball of $X$. Set $\bar{e}=\sum_{i=1}^{m} \lambda_{i} e_{i}$. Then $\sum_{i=1}^{m} \lambda_{i} R_{e_{i}}=R_{\bar{e}}$ and consequently the resolvent average [1] of $A_{e_{1}}, \ldots, A_{e_{m}}$, with parameters $\lambda_{1}, \ldots, \lambda_{m}$, is $A_{\bar{e}}$.

\section{Acknowledgments}

HHB was partially supported by the Natural Sciences and Engineering Research Council of Canada. WMM was partially supported by the Natural Sciences and Engineering Research Council of Canada Postdoctoral Fellowship.

\section{REFERENCES}

[1] S. Bartz, H.H. Bauschke, S.M. Moffat, X. Wang, The resolvent average of monotone operators: dominant and recessive properties, SIAM J. Optim. 26 (2016), 602-634.

[2] H.H. Bauschke, P.L. Combettes, Convex Analysis and Monotone Operator Theory in Hilbert Spaces, Second Edition, Springer, 2017.

[3] H.H. Bauschke, S.M. Moffat, X. Wang, Firmly nonexpansive mappings and maximally monotone operators: correspondence and duality, Set-Valued Var. Anal. 20 (2012), 131-153.

[4] H. Brezis, Operateurs Maximaux Monotones et Semi-Groupes de Contractions dans les Espaces de Hilbert, NorthHolland/Elsevier, 1973.

[5] R.S. Burachik, A.N. Iusem, Set-Valued Mappings and Enlargements of Monotone Operators, Springer-Verlag, 2008.

[6] K. Goebel, W.A. Kirk, Topics in Metric Fixed Point Theory, Cambridge University Press, 1990.

[7] K. Goebel, S. Reich, Uniform Convexity, Hyperbolic Geometry, and Nonexpansive Mappings, Marcel Dekker, 1984.

[8] M. Kac, Statistical Independence in Probability, Analysis and Number Theory, Wiley, 1959.

[9] E. Kopecká, S. Reich, Nonexpansive retracts in Banach spaces, in Fixed Point Theory and its Applications, Banach Center Publications, Vol. 77, 161-174. 2007.

[10] G.J. Minty, Monotone (nonlinear) operators in Hilbert spaces, Duke Math. J. 29 (1962), 341-346.

[11] R.T. Rockafellar, R.J-B. Wets, Variational Analysis, Springer-Verlag, corrected 3rd printing, 2009.

[12] S. Simons, From Hahn-Banach to Monotonicity, Springer-Verlag, 2008.

[13] S. Simons, Minimax and Monotonicity, Springer-Verlag, 1998.

[14] C. Zălinescu, Convex Analysis in General Vector Spaces, World Scientific Publishing, 2002.

[15] E. Zeidler, Nonlinear Functional Analysis and Its Applications I: Fixed Point Theorems, Springer-Verlag, 1993.

[16] E. Zeidler, Nonlinear Functional Analysis and Its Applications II/A: Linear Monotone Operators, Springer-Verlag, 1990.

[17] E. Zeidler, Nonlinear Functional Analysis and Its Applications II/B: Nonlinear Monotone Operators, Springer-Verlag, 1990. 